\title{
Unintentional Weight Loss as the Sole Indication for Colonoscopy is Rarely Associated with Colorectal Cancer
}

\author{
Ian J. Davis, DO, Shannon J. Marek, MD, Subbaramiah Sridhar, MBBS, \\ Thad Wilkins, MD, Sherman M. Chamberlain, MD
}

Introduction: Weight loss is a commonly used indication for colonoscopy.

Methods: This is a prospective case study of colonoscopies from 1998 to 2009. Descriptive statistics were used to evaluate age, sex, colonoscopy indications, and findings. Multiple logistic regression analysis was used to determine the odds of colorectal cancer (CRC) based on age, sex, and weight loss.

Results: We reviewed 6425 colonoscopies. The mean age of patients was 57.4 years $(\mathrm{SD}, \pm \mathbf{1 3 . 5}$ years), and $55 \%$ of patients were women. One hundred thirty-six $(2.1 \%)$ of these had unintentional weight loss; for 32 patients $(0.4 \%)$, unintentional weight loss was the only indication for the procedure. CRC was diagnosed in 116 patients $(1.8 \%)$, but CRC was not detected in any patients for whom unintentional weight loss as the only indication for colonoscopy.

Conclusion: Based on our prospective case study, unintentional weight loss alone was not associated with CRC. (J Am Board Fam Med 2011;24:218-219.)

Keywords: Colonoscopy, Colorectal Cancer, Colorectal Cancer Detection, Colorectal Cancer Symptoms, Weight Loss

Weight loss is used as an indication for colonoscopy. The rationale for this likely is because of the association of weight loss with many types of occult malignancy. One third of elderly patients with unintentional weight loss were diagnosed with malignancy. ${ }^{1-4}$ Gastrointestinal malignancy is the most common type of occult malignancy diagnosed in patients with unintentional weight loss, and colorectal cancer (CRC) ranks third overall among the most common types of cancer in the United States. ${ }^{5}$ In this study we examined the association between unintentional weight loss as a sole indication for colonoscopy and the risk for CRC.

This article was externally peer reviewed.

Submitted 19 July 2010; revised 5 October 2010; accepted 28 October 2010.

From the Department of Internal Medicine (IJD, SJM, SMC); the Section of Gastroenterology and Hepatology (SJM, SS, SMC); and the Department of Family Medicine (TW), Medical College of Georgia-Georgia Health Sciences University, Augusta.

Funding: none.

Conflict of interest: none declared.

Corresponding author: Sherman M. Chamberlain, MD, Department of Internal Medicine, Section of Gastroenterology and Hepatology, Medical College of Georgia, 1120 Fifteenth Street, Augusta, GA 30912 (E-mail: schamberlain@georgiahealth.edu).

\section{Methods}

This was a prospective case study of de-identified data collected by one gastroenterologist (SMC) between 1998 and 2009. The data were collected prospectively and included patients' age, sex, and indications for and findings of the colonoscopy. Findings of CRC and polyps biopsies were confirmed by pathologic results. SAS software version 9.2 (SAS Institute, Inc., Cary, NC) was used for all statistical analyses and an $\alpha$ level of 0.05 was used to assess statistical significance. To examine the relationship of weight loss, age, and sex with CRC, multiple logistic regression was used to calculate an estimate of the odds ratio for each factor. This study was approved by the Human Assurance Committee (institutional review board) at the Medical College of Georgia.

\section{Results}

Data from 6425 colonoscopies were reviewed (mean age of patients, 57.4 years; SD, \pm 13.5 years; $55 \%$ of patients were women). One hundred sixteen patients $(1.8 \%)$ were diagnosed with CRC. Out of all colonoscopies, 136 (2.1\%) had unintentional weight loss as an indication, and only 32 
patients $(0.4 \%)$ had weight loss as the sole indication for the procedure. The remaining 104 patients had unintentional weight loss listed as an indication with other symptom: 32 patients $(0.4 \%)$ had gastrointestinal bleeding or anemia, 42 patients $(0.7 \%)$ had changes in their bowel habits, and 16 patients $(0.2 \%)$ had abdominal pain. Of the 136 patients with unintentional weight loss as an indication for colonoscopy, only $5(0.07 \%)$ were diagnosed with CRC, each of whom had unintentional weight loss and an additional indication for colonoscopy (eg, gastrointestinal bleeding, anemia, or abdominal pain). Weight loss was not significantly associated with CRC $(P=.1637)$. The odds ratio for detecting CRC with weight loss was 1.9 (95\% CI, $0.8-$ 4.8; see Table 1).

\section{Discussion}

In our study, we report a prevalence of unintentional weight loss of $2 \%$, with only 5 cases of CRC detected through colonoscopy. The results of this study demonstrate that weight loss as a sole indication for performing colonoscopy may not be sufficient. Other authors report that the cause for unintentional weight loss can be typically found through the patient's history, physical examination,

Table 1. The Association of Age, Sex, and Weight Loss with Colorectal Cancer

\begin{tabular}{lcr}
\hline & $\begin{array}{c}\text { Colorectal Cancer } \\
\text { (OR [95\% Confidence }\end{array}$ & \multicolumn{1}{c}{$P$} \\
\hline Effect & Limits]) & $<.0001$ \\
Age (years)* & $1.3(1.2,1.4)$ & .1998 \\
Sex (male vs female) & $1.3(0.9,1.8)$ & .1637 \\
\hline
\end{tabular}

*Odds ratio (OR) estimate for 5-year age increments. and routine laboratory testing, with further studies performed as indicated. ${ }^{1,4}$ Routine testing may include a complete blood count, complete metabolic panel, thyroid studies, C-reactive protein analgesia, and a chest radiograph for patients who smoke or who have respiratory symptoms. ${ }^{3}$ This was a single-endoscopist case study, although the information was entered prospectively immediately after the procedure. Furthermore, the amount of weight loss was not quantified or verified by chart audit. We did not include race, marital status, education, or insurance status in our database.

\section{Conclusions}

Unintentional weight loss can be a systemic response to multiple disease processes such as endocrine, gastrointestinal, cardiopulmonary, or rheumatic diseases. Our findings suggest that unintentional weight loss as the only indication for colonoscopy may not be associated with CRC.

\section{References}

1. Hernandez JL, Matorras P, Riancho JA, GonzalezMacias J. Involuntary weight loss without specific symptoms: a clinical prediction score for malignant neoplasm. QJM 2003;96:649-55.

2. Lankisch P, Gerzmann M, Gerzmann JF, Lehnick D. Unintentional weight loss: diagnosis and prognosis. The first prospective follow-up study from a secondary referral centre. J Intern Med 2001;249:41-6.

3. Metalidis C, Knockaert DC, Bobbaers H, Vanderschueren S. Involuntary weight loss. Does a negative baseline evaluation provide adequate reassurance? Eur J Int Med 2008;19:345-9.

4. Vanderschueren S, Geens E, Knockaert D, Bobbaers $\mathrm{H}$. The diagnostic spectrum of unintentional weight loss. Eur J Int Med 2005;16:160-4.

5. Jemal A, Siegel R, Ward E, Hao Y, Xu J, Thun MJ. Cancer statistics, 2009. CA Cancer J Clin 2009;59: 225-49. 